\title{
Dissolved biochar promoted photodegradation of tetracycline in aqueous environment
}

\author{
Chenjing $\mathrm{Chu}^{1,2}$, Mengying Shao ${ }^{1,2}$, Xiao Wang ${ }^{1,2, *}$ \\ ${ }^{1}$ Institute of Coastal Environmental Pollution Control, Key Laboratory of Marine Environment and Ecology, Ministry of Education, \\ Ocean University of China, Qingdao 266100, China \\ ${ }^{2}$ College of Environmental Science and Engineering, Ocean University of China, Qingdao 266100, China
}

\begin{abstract}
As one of the frequently detected antibiotics in aqueous environment, the environmental behaviour of tetracycline has received increasing attention. Dissolved biochar (DBC) is an essential component of dissolved organic matter (DOM) in water enviroment. However, the effect of DBC on the photodegradation of tetracycline was unclear. This study investigated the impact of DBC on the photodegradation of tetracycline in aqueous solution under light illumination. The main components of DBC were humic acid-like and fulvic acid-like, accounting for $78.2 \%$ of the total components. The DBC surface had more oxygen-containing functional groups and aromatic functional groups. DBC increased the photodegradation of tetracyline by $46.9 \%$, which was related to the component and structure of $\mathrm{DBC}$. These findings would provid theoretical support for the environmental behaviour of DBC and tetracycline in aqueous environment.
\end{abstract}

\section{Introduction}

Due to the abuse of antibiotics, most of them cannot be absorbed and metabolized and finally discharged into the environment, resulting in a large amount of antibiotics remaining in the environment. The detection frequency and content of tetracycline in all antibiotics were relatively high, and its fate in the environment has attracted the attention of scholars. In the natural environment, indirect photolysis was the main way to degrade tetracycline. Indirect photolysis was a photochemical reaction caused by the addition of photocatalyst or photosensitizer. Under light illumination, the photosensitizer transmits electrons to the compound to change its stability and accelerate its photolysis[1]. Dissolved organic matter (DOM) was an important photosensitizer in the environment [2]. Natural DOM can produce three-state excited state DOM $\left({ }^{3} \mathrm{DOM}^{*}\right)$, singlet oxygen, superoxide anion, hydrogen peroxide and hydroxyl radicals and other strong oxidizing substances, which promoted the photochemical conversion of DOM itself and organic pollutants [3].

Biochar was a carbonaceous porous material obtained by incomplete combustion of biomass under anaerobic or anaerobic conditions [4]. It has been widely used in agriculture as a soil amendment to improve agricultural and environmental benefits. In the past few years, dissolved Biochar (DBC) has aroused the research interest of scholars at home and abroad. DBC was usually defined as the organic carbon component smaller than $0.45 \mu \mathrm{m}$. DBC included the dissolved organic matter of $\mathrm{BC}$ and some particles with a particle size smaller than $0.45 \mu \mathrm{m}$. Compared with BC, DBC has stronger hydrophilicity, which was related to its abundant polar functional groups, such as aliphatic carbon and carboxyl carbon. At the same time, DBC also has fused-ring aromatic hydrocarbons and a higher proportion of oxygen-containing functional groups [5]. However, whether DBC could promote the photodegradation of tetracycline, the proportion and mechanism of promoting photolysis are still unclear. Because of its higher content of photosensitive components (humic acid-like and fulvic acid-like), the effect of DBC on the photolysis of tetracycline and its mechanism was explored through light experiments. Therefore, this word explored the properties of DBC and the photodegradation rate of tetracycline after adding DBC.

\section{Methods and materials}

\subsection{Experimental materials}

The biomass in this paper was selected pepper straw, which was cleaned and dried for preservation. The preparation of biochar adopts oxygen-limited slow pyrolysis method [5]. After the nitrogen gas was introduced for $30 \mathrm{~min}$, the temperature was increased at a rate of $10^{\circ} \mathrm{C} \mathrm{min}^{-1}$, and the temperature was increased to $450{ }^{\circ} \mathrm{C}$, respectively. After cooling, the $\mathrm{BC}$ were removed from the furnace and ground to pass a $0.125-\mathrm{mm}$ sieve, labelled DBC450. Two grams of $\mathrm{BC}$ and $20 \mathrm{~mL}$ of deionized water were shaken at $130 \mathrm{rpm}$ in a shaking incubator at room temperature for $12 \mathrm{~h}$, and then the $\mathrm{BC}$ suspension was passed through a $0.45 \mu \mathrm{m}$ cellulose acetate filter membrane (Jinteng, China) [6]. After

\footnotetext{
* Corresponding author: wangxiao5120@ouc.edu.cn
} 
filtration, the residue remaining on the membrane was collected and subjected to $100 \mathrm{~W}$ ultrasonic treatment for 30 minutes, and then a new round of filtration ultrasonic treatment was performed to obtain a total of $60 \mathrm{~mL}$ of DBC. DBC was stored in a refrigerator at $-20^{\circ} \mathrm{C}$ in the dark. Tetracycline was purchased from Sangon Biotech Co., China. Molecular structure of tetracycline is presented in Fig. 1.

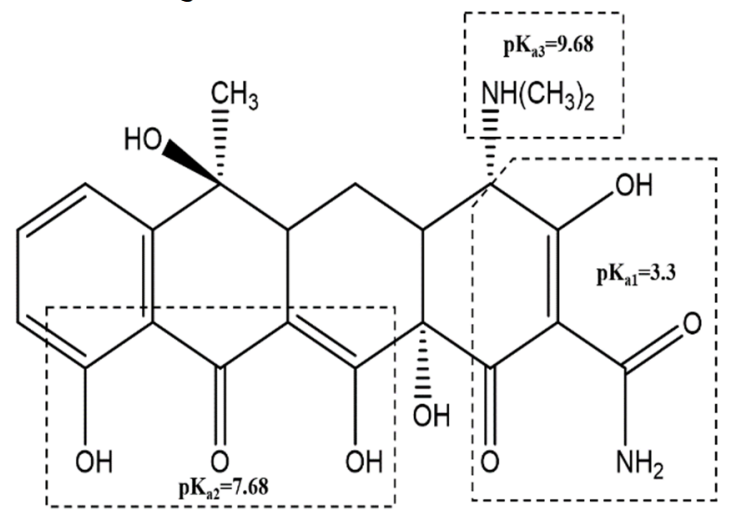

Fig. 1. Molecular structure of tetracycline [7].

\subsection{Characterization}

DBC450 were characterized by $\mathrm{pH}$ meterl (AB150, Thermo Fisher Scientific, U.S.A), Total organic carbon (TOC) analyzer (TOC-VCPN, Shimadzu, Janpa), threedimension excitation emission matrix fluorescence spectroscopy (3D-EEM) (F-4600, Hitachi HighTechnology Corp, Japan), ultraviolet-visible (UV-vis) (UV-9000S, Metash, China) absorption and Fourier transform infrared (FTIR) (Spectrum Two, PerkinElmer, UK). TOC analyzer was used to determine the total carbon content of DBC, and standard curve of potassium hydrogen phthalate was selected. Each sample was tested 3 times in parallel. After diluting DBC to $100 \mathrm{mg} \mathrm{L}^{-1}, 2$ $\mathrm{mL}$ of $\mathrm{DBC}$ was taken out for UV-vis scanning, the scanning wavelength was $900-100 \mathrm{~nm}$; also $3 \mathrm{~mL}$ of DBC for 3D-EEM, the emission scanning was in the 200-550 $\mathrm{nm}$ wavelength range. It was performed in $1 \mathrm{~nm}$ increments, and the excitation wavelength was performed in $1 \mathrm{~nm}$ increments at 220-450 nm [5]. $\mathrm{KBr}$ was added to $100 \mathrm{mg} \mathrm{L}{ }^{-1} \mathrm{DBC}$, mixed and freeze-dried at $-70^{\circ} \mathrm{C}$. The dried mixture was ground into sheets for FTIR, and the scanning area was $4000-500 \mathrm{~cm}^{-1}[8]$.

\subsection{Photolysis experiment}

In the photolysis experiment, concentrations of DBC and tetracycline were 10 and $20 \mathrm{mg} \mathrm{L}^{-1}$, respectively, and a blank control group without any treatment was set. The background solution used in the experimental system was deionized water. Placed the treatment group in the photoreactor (BL-GHX, Shanghai Birang, China), and kept it for 10 minutes to stabilize the light intensity at 30 $\mathrm{W} \mathrm{m} \mathrm{m}^{-2}$. The photoreactor was equipped with a $290-400$ $\mathrm{nm}$ filter (include UVA and UVB), and the reactor was rotated at a constant speed to ensure that each quartz tube can be illuminated evenly [9]. Performed the same treatment under dark conditions. Samples were taken at 0,
10, 20, 40, and 60 minutes of the reaction. Each time, 600 $\mu \mathrm{L}$ of the reaction solution was taken out and filtered with a $0.22 \mu \mathrm{m}$ water-based filter membrane. The detection method of tetracycline was as follows: a chromatographic column of $4.5 \times 250 \mathrm{~mm}$ Agilent C18 was selected; filtered with a concentration of $1.2607 \mathrm{~g} \mathrm{~L}^{-1}$ oxalic acid solution and chromatographically pure acetonitrile with a $0.22 \mu \mathrm{m}$ filter membrane and ultrasonicated for $30 \mathrm{~min}$; acetonitrile in the instrument: oxalic acid solution $=25: 75$ $(\mathrm{V} / \mathrm{V})$ as the mobile phase; ultraviolet detector (VWD) wavelength as $355 \mathrm{~nm}$; mobile phase flow rate as $1 \mathrm{~mL}$ $\mathrm{min}^{-1}$, and sample injection volume as $20 \mu \mathrm{L}$ [10].

\section{Results and discussion}

\subsection{Characteristics of DBC450}

In order to explore the composition of $\mathrm{DBC}, \mathrm{DBC}$ was charactered by 3D-EEM. The 3D-EEM spectrum of DBC was shown in Figure 2a. After identifying with the literature, the EEM profile was divided into 5 components [11]. Component I corresponds to the ultraviolet fluorescence of tyrosine-like protein substances, which is composed of Ex: 200-250nm and Em:280-330 nm excitation wavelength range; component II corresponds to the ultraviolet fluorescence of tryptophan-like protein substances and consists of wavelength ranges (Ex: 200$250 \mathrm{~nm}$; Em: $330-380 \mathrm{~nm}$ ); component III corresponds to the ultraviolet fluorescence of fulvic acid-like substances, and consists of wavelength ranges (Ex: 200-250 nm; Em: 380-550 nm); component IV corresponds to the ultraviolet fluorescence of soluble microbial metabolites, consisting of wavelength range (Ex: 200-250 nm; Em: 330-380 nm); component V corresponds to the ultraviolet fluorescence of fulvic acid-like substances and consists of wavelength range (Ex: 250-400 nm; Em: 380-550 nm). The proportions of each component in DBC were $3.07 \%$, $7.74 \%, 26.0 \%, 11.0 \%$ and $52.1 \%$. The main components of DBC were humic acid-like and fulvic acid-like, accounting for $78.2 \%$ of the total components. These substances have high aromaticity and electron transport capacity. More importantly, humic and fulvic acids were photosensitive.

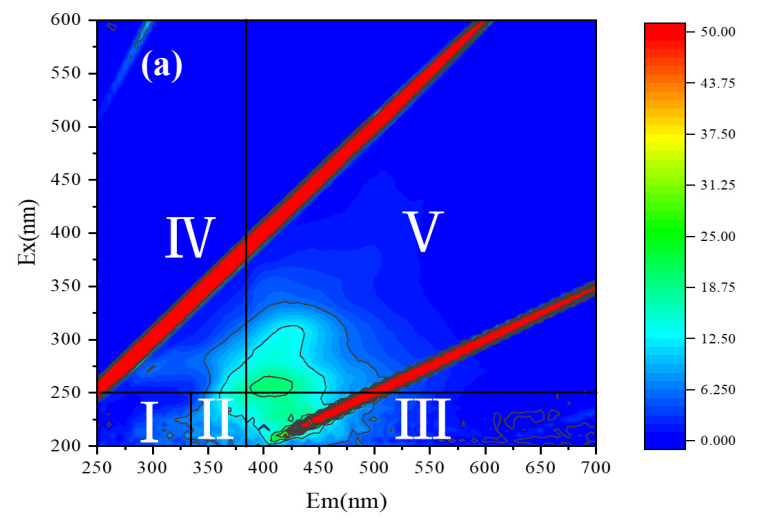



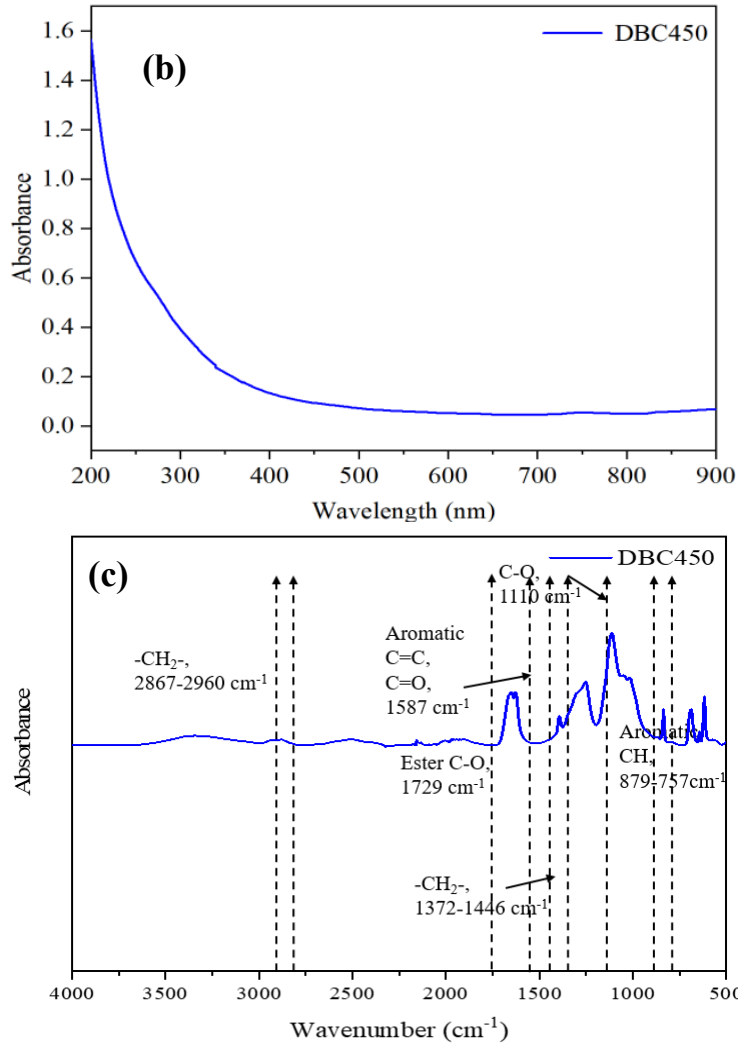

Fig. 2. EEM (a), UV-vis (b) and FTIR spectrogram (c) of DBC450.

In order to explore the structure of the $\mathrm{DBC}, \mathrm{DBC}$ was charactere by UV-vis. The UV-vis spectrum of DBC was shown in Figure 2b. DBC has a large absorption peak at low wavelength. As the wave number increased, the absorbance of DBC dropped sharply. This was because DBC was mainly aromatic substances, and its main absorption peaks were all in the ultraviolet region, which was similar to the characteristic spectrum of humus [12]. The UV254 of DBC is less than 3, indicating that there are many hydrophilic substances in DBC. Similar results can be obtained by E2/E3 (the ratio of the absorbance of $\mathrm{DBC}$ at 254 and $365 \mathrm{~nm})$. The absorbance (E4/E6) of the $\mathrm{DBC}$ at 465 to $665 \mathrm{~nm}$ was inversely proportional to the aromaticity, indicating that the $\mathrm{DBC}$ contains aromatic components.

In order to explore the surface functional groups of DBC, DBC was charactered by FTIR. The FTIR spectrum of DBC was shown Fig. 2c, and the main spectral bands were classified. DBC contained a large number of oxygen-containing functional groups, which means that DBC had good electron transport capacity and more adsorption sites when adsorption with organic matter occurs. The presence of Ester functional groups (Ester C$\mathrm{O}, 1729 \mathrm{~cm}^{-1}$ ) in $\mathrm{DBC}$ reduced the $\mathrm{pH}$ of the solution during hydrolysis, and the presence of aliphatic methylene $\left(-\mathrm{CH}_{2-}, 1372-446 \mathrm{~cm}^{-1}, 2867-2960 \mathrm{~cm}^{-1}\right)$ in DBC was similar to the results reported in the literature [5]. At the same time, aromatic hydrocarbon (Aromatic C-H, 757$879 \mathrm{~cm}^{-1}$ ) and aromatic functional groups gradually increased (Aromatic $\mathrm{C}=\mathrm{O}, \mathrm{C}=\mathrm{C}, 1587 \mathrm{~cm}^{-1}$ ), showing a similar pattern to EEM of DBC.

\subsection{Effect of DBC on the photodegradation of tetracycline in aqueous environment}

The results of the effect of DBC on photodegradation of tetracycline were shown in Fig. 3. Under dark controls, $\mathrm{DBC}$ had no effect on concentration of tetracycline. This was because DBC did not degrade tetracycline under dark conditions. There was no photodegradation of tetracycline in the control group. When DBC was added, the photolysis of tetracycline was significantly improved, and the effect of DBC on the photolysis of tetracycline in 1 hour was $46.9 \%$. Under light illumination, DBC will produce active intermediates with oxidation capacity. DBC produced ROS due to its highly similar molecular structure with humic acid and the number of oxygencontaining functional groups. At the same time, DBC has a high aromatics, which means that aromatic substances in DBC were replaced by oxygen. Previous studies have shown a positive correlation between the surface ROS stable state concentration and the degradation rate of organic matter. Under light illumination, electrons were transferred from tetracycline to ROS, forming phenoxy free radicals and generating self-coupling, and further oxidation occurred under the action of ROS, which leaded to the destruction of the structure of tetracycline and loss of toxicity [6].

In this study, the background solution after adding DBC and tetracycline was alkaline $(\mathrm{pH}=10.4)$. Under this condition, the tetracycline was ionized and presented a negative charge state $\left(\mathrm{H}_{2} \mathrm{TC}^{-}\right) . \mathrm{H}_{2} \mathrm{TC}^{-}$has a higher electron density than tetracycline. Therefore, it was more susceptible to attack by ROS, which leaded to a higher photodegradation rate of tetracycline when the $\mathrm{pH}$ of the solution was higher [13]. At the same time, the humic acid-like and protein-like components in DBC formed a complex with tetracycline, and the increasing ROS under light illumination contact tetracycline directly. The complex between DBC and tetracycline made tetracycline more oxidized easily, which may be another reason why DBC accelerated the photolysis of tetracycline.

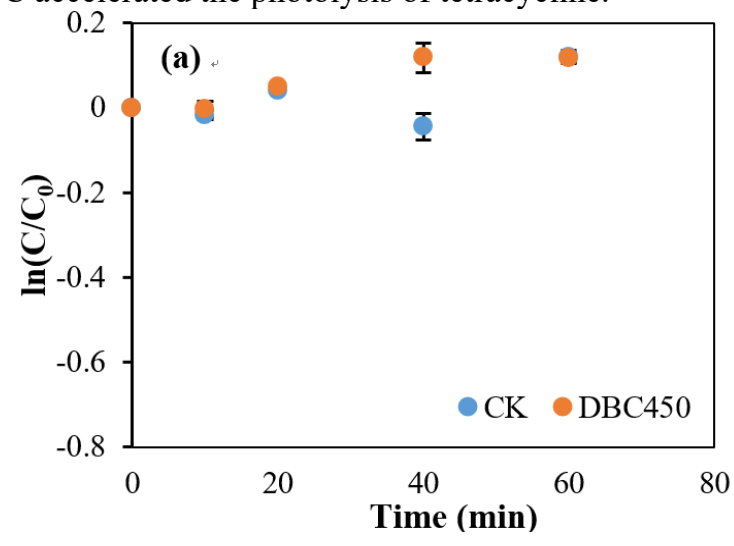




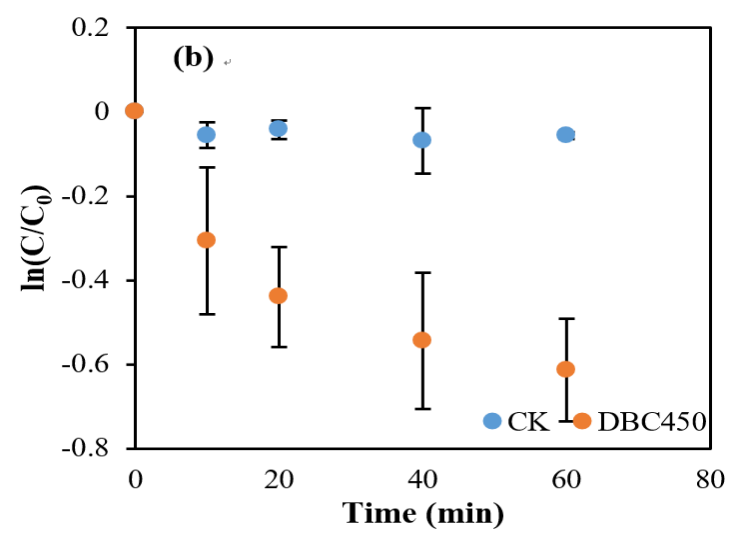

Fig. 3. Change of tetracycline concentration with or without DBC under dark controls (a) and light illumination (b). Concentrations of DBC and tetracycline were 10 and $20 \mathrm{mg} \mathrm{L}$ ${ }^{1}$, respectively.

\section{Conclusions}

In this study, the main components of DBC were humic acid-like and fulvic acid-like, accounting for $78.2 \%$ of the total components. The DBC surface had more oxygencontaining functional groups and aromatic functional groups. After adding DBC, the photodegradation rate of tetracycline increased by $46.9 \%$. This was because ROS mediated the formation of phenoxy radicals under light illumination and ultimately accelerated the photodegradation of tetracycline. It was more susceptible to attack by ROS, which leaded to a higher photodegradation rate of tetracycline when the solution was alkaline. And the oxygen-containing functional groups and aromatic substances in DBC complexed with tetracycline to bind DBC and tetracycline tightly. These reasons improved the reaction efficiency between DBC and tetracycline under light irradiation, and finally enhanced the photodegradation rate of tetracycline. These findings would provide theoretical support for the environmental behaviour of DBC and tetracycline in the aqueous environment.

\section{Acknowledgments}

This study was supported by Shandong Key Research and Development Program-Science and Technology Innovation Project (2018CXGC0304) and Shandong Province Natural Science Foundation (ZR2019MD017).

\section{References}

1. Y.J. Dai, M. Liu, J.J. Li, S.S. Yang, Q.Y. Sun, W.S. Wang, L. Lu, K.X Zhang, J.Y. Xu, W.L. Zheng, Z.Y. Hu, Y.H. Yang, Y.W. Gao, Z.H. Liu, Sep. Sci Technol 55, 1005-1021 (2019).

2. O.M.S. Filipe, E.B.H. Santos, M. Otero, E.A.C. Gonçalves, M.G.P.M.S. Neves, J. Hazard. Mater 385, 121523 (2020).

3. M. Li, F.X. Bao, Y. Zhang, H. Sheng, C.C. Chen, J.C. Zhao, Environ. Sci. Technol 53, 12311-12319 (2019).
4. X. Xiao, B.L. Chen, Z.M. Chen, L.Z. Zhu, J.L. Schnoor, Environ. Sci. Technol 52, 5027-5047 (2018).

5. X.L. Qu, H.Y. Fu, J.D. Mao, Y. Ran, D.N. Zhang, D.Q. Zhu, Carbon 96, 759-767 (2016).

6. Z.C. Zhou, B.N. Chen, X.L. Qu, H.Y. Fu, D.Q. Zhu, Environ. Sci. Technol 52, 10391-10399 (2018).

7. Y.K. Lee, J. Hur, Water Res 187, 116426 (2020).

8. S.Q. Wu, H.Y. Hu, Y. Lin, J.L. Zhang, Y.H. Hu, Chem. Eng. J 382,122842 (2020).

9. X. Zhang, J. Li, W.Y. Fan, M.C. Yao, L. Yuan, G.P. Sheng, Environ. Sci. Technol 53, 10732-10740 (2019).

10. M.M. Wang, L.S. Ren, D.Y. Wang, Z.S. Cai, X.F. Xia, A.Z. Ding, J. Environ. Sci 79, 91-99 (2019).

11. M. Park, S.A. Snyder, Chemosphere 193, 530-537 (2018).

12. J. Chen, B. Gu, E.J. LeBoeuf, H. Pan, S. Dai, Chemosphere 48, 59-68 (2002).

13. C.J. Li, D.H. Zhang, J.L. Peng, X.G. Li, J Photoch. Photobio. A 356, 239-247(2018). 